\title{
Effectiveness of a Pulmonary Rehabilitation Program on Changes in Heart Rate Variability and Physical Performance in Chronic Obstructive Pulmonary Disease
}

\section{Szucs $\mathbf{B}^{1}$, Petrekanits $\mathbf{M}^{2}$ and Varga $\mathbf{J}^{3^{*}}$}

${ }^{1}$ Pharma Flight Research and Training Center, Debrecen, Hungary

${ }^{2}$ Institute of Exercise Physiology and Sport Medicine, University of Physical Education, Budapest, Hungary

${ }^{3}$ Department of Pulmonary Rehabilitation, National Koranyi Institute for Pulmonology, Budapest, Hungary

\begin{abstract}
Changes in autonomic regulation are evaluated in COPD patients by using heart rate variability (HRV) in rehabilitation. Our aim was to evaluate the presence of autonomic dysfunction and to assess the effect of a rehabilitation program.

$R-R$ intervals were measured for 6 minutes in 36 patients ( 16 female; 20 male) before and after an inpatient pulmonary rehabilitation program that included 30 minutes of respiratory training, chest wall mobilization, learning controlled breathing techniques, inhalation, expectoration and personalized training. Ectopic beats were eliminated. Parameters used: minimal pulse (p.min), average pulse (p.avg), maximal pulse (p.max), maximum-minimum pulse difference (p.max-p.min). Long-term continuous RR intervals (stda), standard deviation of instantaneous beat-to-beat variability (stdb), the number of pairs of adjacent $N N$ intervals differing by more than $50 \mathrm{~ms}$ divided by the total number of all NN intervals (pNN50). Spectral analysis provided the low-frequency/high-frequency ratio (LF/HF).

HRV showed decreased p.max-p.min (15.78 \pm 9.2 bpm), depressed dynamics (stda: $39.63 \pm 33.5$; stdb: $22.72 \pm$ 35.84 ) with sympathetic overload (pNN50:3.17 \pm 5.24 , LF/HF: $169.52 \pm 208.83$ ), heavy parasympathetic modulation (pNN50:5.51 \pm 5.59 , LF/HF: $27.28 \pm 13.12$ ) in severe COPD patients. Rehabilitation resulted in lowered p.min-p.max $(12.5 \pm 9.01 \mathrm{bpm})$, overdepressed dynamics (stda: $34.56 \pm 35.97$; stdb: $20.88 \pm 41.5)$ strong sympathetic overload (pNN50:3.33 \pm 6.76 , LF/HF: $252.01 \pm 351.16$ ).

Patients showed abnormal physiological response in resting autonomic regulation. The rehabilitation resulted in improvement in overall status, autonomic balance.
\end{abstract}

Keywords: Chronic obstructive pulmonary disease; Heart rate variability; Pulmonary rehabilitation; Cardiovascular function; Sympathetic and parasympathetic nervous system

\section{Introduction}

Chronic obstructive pulmonary disease (COPD) is a complex and heterogeneous clinical syndrome found in $6-8 \%$ of the population [1]. COPD patients experience functional and structural changes of the respiratory system that deeply influence cardiovascular function [2]. Several factors, including abnormal autonomic control of cardiopulmonary function, may contribute to the development of arrhythmias in these patients [3-7]. Determination of autonomic balance may be important in understanding the pathophysiology of COPD and might be useful clinically in the treatment of patients with COPD [8].

The application of heart rate variability (HRV) to assess the risk of sudden cardiac death and diabetic neuropathy is well known $[9,10]$. The sympathetic and parasympathetic (autonomic) nervous systems innervate the heart and regulate the heart rate (HR). As illustrated in Figure 1 the HRV represents a physiological phenomenon that may be monitored and analyzed to determine the state of the nervous system that controls the heart [11]. HRV data can be collected in relatively simple devices such as modern wrist computers [12-14]. although autonomic control of the cardiovascular system is also affected by baroreceptors, chemoreceptors, muscle afferents, local tissue metabolism, and circulating hormones (Figure 2) [15]. Critical adjustments are continually made to the cardiovascular system to meet the diverse demands of the musculature and heart [16,17]. These dynamic adjustments in cardiac and peripheral vascular control, including their regulation by the autonomic nervous system (ANS), occur in part as a response to rapid changes in heart rate and blood

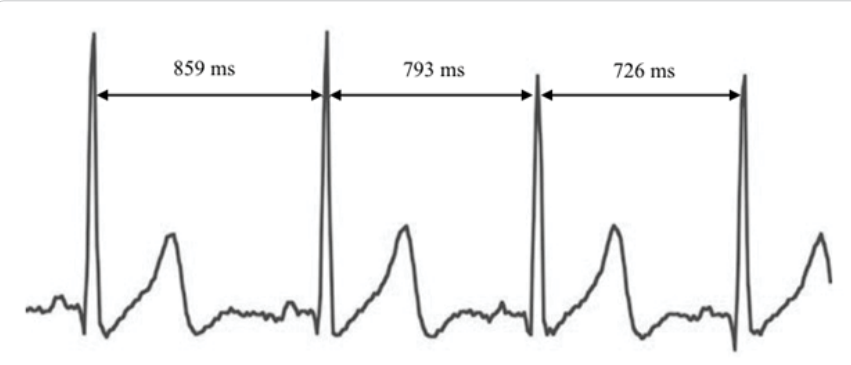

Figure 1: $R-R$ variants of $R-R$ distance for calculation of heart rate variability (HRV).

pressure. Though there are some reports on changes in time-domain and frequency-domain heart rate variability in COPD patients, the information on $\mathrm{HRV}$ in patients with COPD has so far been conflicting [18-21].

*Corresponding author: Janos Varga, Department of Pulmonary Rehabilitation, National Koranyi Institute for Pulmonology, Budapest, Hungary, Tel: +3613913374; Fax: +3613913285; E-mail: varga@koranyi.hu

Received September 25, 2018; Accepted September 28, 2018; Published October 04, 2018

Citation: Szucs B, Petrekanits M, Varga J (2018) Effectiveness of a Pulmonary Rehabilitation Program on Changes in Heart Rate Variability and Physical Performance in Chronic Obstructive Pulmonary Disease. J Pulm Respir Med 8: 474. doi: 10.4172/2161-105X.1000474

Copyright: (2018 Szucs B, et al. This is an open-access article distributed under the terms of the Creative Commons Attribution License, which permits unrestricted use, distribution, and reproduction in any medium, provided the original author and source are credited. 


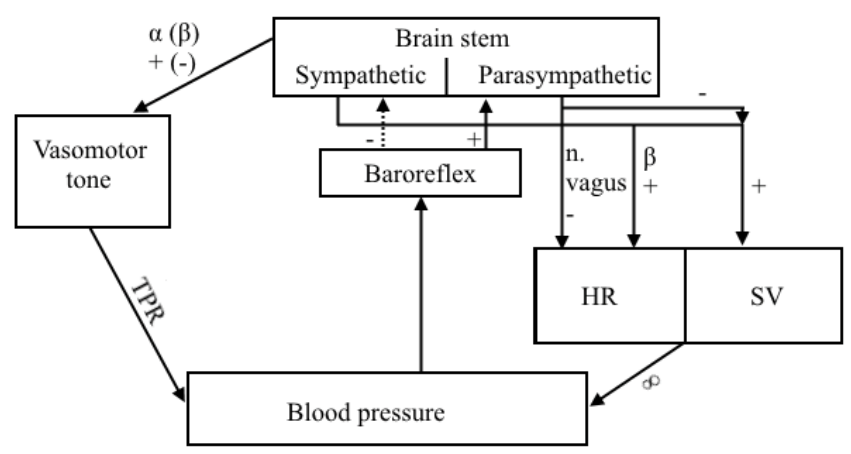

Figure 2: This model of HRV control illustrates independent actions of the vagal, $\alpha$ - and $\beta$ - sympathetic system. The actions of these systems can be assessed by measuring heart rate variability, blood pressure variability, and the baroreflex mechanism. The parasympathetic nervous system is responsible for the bradycardia accompanying baroreceptor stimulation, and for the tachycardia accompanying baroreceptor deactivation, with the sympathetic nervous system also playing a minor role.

Exercise training reduces the possibility of sudden cardiac death and decreases cardiovascular mortality [22]. Regular training and exercise play an important role in COPD rehabilitation. The effectiveness of pulmonary rehabilitation can be monitored by measuring HRV and cardiac function because exercise training is capable of modifying the autonomic balance. Since the complex mechanism of HRV changes are not totally clear, experimental studies are still needed to explain specific changes in HRV [23].

The aim of this study was to evaluate the presence of autonomic dysfunction in patients with COPD by HRV and to assess the effect of a four-week rehabilitation program.

\section{Materials and Methods}

\section{Study subjects}

36 patients with COPD (age: $65.4 \pm 7.4$ years (51-78); BMI: 26 $\pm 5 \mathrm{~kg} / \mathrm{m}^{2}$, male:female: $\left.20: 16\right)$ participated in this study. All of the patients provided written consent for the study in the Department of Pulmonary Rehabilitation. The study protocol was approved by the Ethical Committee of the National Koranyi Institute for Pulmonology with 25/2017 registration number. It was an observational study using the general management of the patient; it was a non-interventional study. Patients' characteristics are presented in Table 1.

\section{Pulmonary function}

According to ATS/ERS guidelines all patients underwent a postbronchodilator pulmonary function testing (Vmax 229 and Autobox 6200 , Sensormedics), including spirometry measurements [24]. COPD

\begin{tabular}{|c|c|}
\hline \multicolumn{2}{|c|}{ Characteristics (n=36) } \\
\hline Age (years) & $65.47 \pm 7.39$ \\
\hline Male: female & $20: 16$ \\
\hline BMI $\left(\mathrm{kg} / \mathrm{m}^{2}\right)$ & $27.99 \pm 6.98$ \\
\hline $\mathrm{FEV}_{1}(\% \mathrm{pred})$ & $45.43 \pm 20.2$ \\
\hline Hypertension & $32: 36$ \\
\hline Diabetes & $11: 30$ \\
\hline Atherosclerosis & $27: 36$ \\
\hline Pulmonary hypertension & $9: 36$ \\
\hline BMl: body mass index; FEV : forced expiratory volume in the first second
\end{tabular}

Table 1: Patients' characteristics. patients inhaled $400 \mu \mathrm{g}$ salbutamol 20 minutes before testing.

\section{Measurements}

6 minutes walking distance: The 6MWD was measured at the corridor of our department. Before, during and after walking, oxygen saturation and heart rate were measured, and a modified Borg-scale was evaluated. The speed of walking was as fast as possible [25]. The 6MWD was made according to the international guideline [26].

Chest wall expansion: Chest wall expansion (CWE) means the difference of chest circumferences between deep inspiration and expiration. It is measured at the level of processus xypho ideus [27].

Maximal inspiratory pressure: To evaluate MIP we used a special digital instrument. Power Breathe K1 (POWERbreathe International Limited). The calculation of diaphragmatic force was based on the patient's height, weight,age and sex (very poor, poor, average, fair, good, very good). Patients were asked to inhale suddenly with maximal force after a maximal exhalation [28].

Breath holding time: Breath holding time (BHT) displays the severity of COPD. After a maximal inhalation the subjects were asked to hold the breath as long as possible with closed nose and mouth without inhalation [29].

Grip strength measurement: Kern handgrip dynamometer (2016 Kern \& Sohn GmbH-Germany) was used to identify the peripheral muscles force [30].

\section{Protocol of resting HRV evaluation}

During our measurements R-R intervals for autonomic regulation tendencies were measured for 6 minutes in a supine position by Polar H1 sensors and Polar Precision Performance 2.0 software (Polar Electro. Finland). The software enabled the visualization of HR and the extraction of a cardiac period (R-R interval) file in "txt" format. Following digital filtering complemented with manual filtering for the elimination of premature ectopic beats and artifacts, at least $256 \mathrm{R}-\mathrm{R}$ intervals were used for the data analysis, and all arrhythmic beats were excluded from data evaluation. Simple HR data was complemented with an HRV and one lead ECG measurement device iQRS (IQRS Hungary). Measurement frequency was $1 \mathrm{kHz}$. A standardized protocol was used; the measurements were noninvasive and performed at the same time period of the day at the National Koranyi Institute for Pulmonology. We performed our resting evaluations on the first and last day of the rehabilitation. Patients were asked not to do any physical activity on days of measurements, the assessing room was separated. quiet and temperature-controlled (maintained around $24^{\circ} \mathrm{C}$ ). The subjects had rested quietly in a supine position during the measurements.

\section{Parameters of HRV measurements}

The most commonly used HRV parameters in ANS evaluation are the frequency-domain, time-domain, and Poincaré plot parameters. While the nomenclature of the different measurements is complex, they represent different ways to look at the variability and distribution of the heart rate over time.

The following time domain parameters were used: minimal pulse (p.min), average pulse (p.avg), maximal pulse (p.max) of the 6 minutes resting measurements, and maximum minimum pulse difference (p.max-p.min).

For HRV analysis in the frequency domain; we used standard deviation of the long-term continuous RR intervals (stda), standard deviation of instantaneous beat-to-beat variability (stdb), the number 
Citation: Szucs B, Petrekanits M, Varga J (2018) Effectiveness of a Pulmonary Rehabilitation Program on Changes in Heart Rate Variability and Physical Performance in Chronic Obstructive Pulmonary Disease. J Pulm Respir Med 8: 474. doi: 10.4172/2161-105X.1000474

Page 3 of 5

of pairs of adjacent $\mathrm{NN}$ intervals differing by more than $50 \mathrm{~ms}$ divided by the total number of all NN intervals (pNN50) [15,23]. Spectral analysis provided the low-frequency/high-frequency ratio (LF/HF).

The Poincaré plot is a map of points in Cartesian coordinates that is constructed from the values of the RR intervals. Each point is represented on the $\mathrm{x}$-axis by the previous normal $\mathrm{RR}$ interval and on the $y$-axis by the following RR interval [31].

\section{Personalized training program}

Our pulmonary rehabilitation program was an inpatient program; included 30 minutes of respiratory training in the morning, chest wall mobilization, learning-controlled breathing techniques, inhalation, expectoration, improving the overall psychological condition. smoking cessation and personalized training. Patients participated in an individualized continuous or interval type of cycle and/or treadmill training for 10-30 minutes, 2-3 times a day for the whole duration of their hospitalization. The duration of the rehabilitation program was 4 weeks. The intensity of the training was progressive from $60-80 \%$ of peak work rate based on maintaining Borg dyspnea scale breathlessness and leg fatigue both on grade No. 7. The training method was very effective and demanding for the patients; it is equivalent of 6-8 weeks of regular training $[32,33]$.

\section{Statistical analysis}

Patient characteristics, lung function, HRV analysis, resting and exercise functional variables were compared by a paired $t$-test, a nonparametric sign test and a Wilcoxon test. Significance was accepted at the $\mathrm{p}<0.05$ level. The distribution around the mean was expressed as \pm SD in tables. Scatterplot distribution was observed in figures. Distributions were tested for normality by the Kolmogorov-Smirnov test.

\section{Results}

Functional and quality of life parameters are presented before and after the rehabilitation program in Table 2. Pulmonary function did not show improvement after the rehabilitation period (Table 2). Significant changes were detected in MIP, CWE, BHT, GS, 6MWD, CAT, mMRC (Table 2). The clinically significant difference was accepted at $\mathrm{p}<0.05$ level (Table 2).

Eleven patients had severe arrhythmias ( 8 males. 3 females). and the ectopic beats were left out of the analysis. After the four-week rehabilitation, the ectopic beats ceased in all but 4 patients $(3$ males. 1 female).

Minimal, average and maximal pulse decreased, as did maximum

\begin{tabular}{|c|c|c|c|}
\hline Parameter & Before Treatment & After Treatment & p-value \\
\hline FEV $_{1}(\mathrm{I})$ & $44.00 \pm 17.00$ & $45.48 \pm 17.05$ & n.s. \\
\hline FVC $(\mathrm{I})$ & $73.59 \pm 17.04$ & $76.00 \pm 13.84$ & n.s. \\
\hline mMRC & $1.97 \pm 0.61$ & $1.55 \pm 0.56$ & $<0.01$ \\
\hline MIP $\left(\mathrm{cm} \mathrm{H}_{2} \mathrm{O}\right)$ & $60.38 \pm 16.74$ & $69.17 \pm 15.34$ & $<0.001$ \\
\hline CWE $(\mathrm{cm})$ & $3.31 \pm 1.72$ & $4.86 \pm 2.17$ & $<0.001$ \\
\hline BHT $(\mathrm{sec})$ & $28.45 \pm 9.94$ & $35.69 \pm 12.85$ & $<0.001$ \\
\hline GS $(\mathrm{kg})$ & $26.60 \pm 9.92$ & $28.89 \pm 10.01$ & $<0.001$ \\
\hline 6MWD $(\mathrm{m})$ & $347.45 \pm 89.96$ & $403.62 \pm 112.07$ & $<0.001$ \\
\hline CAT & $15.86 \pm 8.07$ & $10.24 \pm 6.16$ & $<0.001$ \\
\hline
\end{tabular}

$\mathrm{FEV}_{1}$ : forced expiratory volume in the first second; FVC: forced vital capacity; mMRC: modified Medical Research Council Dyspnoea Scale; MIP: maximal inspiratory pressure; CWE: chest wall expansion; BHT: breath holding time; GS: grip strength; 6MWD: 6-minute walking distance; CAT: COPD assessment test

Table 2: Functional and quality of life marker parameters.

\begin{tabular}{|c|c|c|c|}
\hline Parameter & Before Treatment & After Treatment & p-value \\
\hline p. $\min (\mathrm{bpm})$ & $68.86 \pm 15.53$ & $66.53 \pm 13.52$ & n.s. \\
\hline p. avg (bpm) & $76.11 \pm 14.26$ & $73.06 \pm 13.04$ & n.s. \\
\hline p. $\max (\mathrm{bpm})$ & $84.5 \pm 14.22$ & $79.31 \pm 13.46$ & n.s. \\
\hline p. max- p. $\min (\mathrm{bpm})$ & $15.78 \pm 9.2$ & $12.5 \pm 9.01$ & $<0.05$ \\
\hline Stda & $39.63 \pm 33.5$ & $34.56 \pm 35.97$ & $<0.05$ \\
\hline Stdb & $22.72 \pm 35.84$ & $20.88 \pm 41.5$ & n.s. \\
\hline pNN50 (\%) & $3.17 \pm 5.24$ & $3.33 \pm 6.76$ & n.s. \\
\hline LF/HF & $169.52 \pm 208.83$ & $252.01 \pm 351.16$ & n.s. \\
\hline
\end{tabular}

p. min: minimal pulse; p. avg: average pulse; p. max: maximal pulse; $p$. max- $p$. min: maximal-minimal pulse difference; stda: standard deviation of the long-term continuous RR intervals; stdb: standard deviation of instantaneous beat-to-beat variability; pNN50: the number of pairs of adjacent NN intervals differing by more than 50 ms divided by the total number of all NN intervals; LF/HF: spectral analysis of the low-frequency/high-frequency ratio

Table 3: HRV results.

minimum pulse difference. These changes showed only a marginal improvement. but significant increment was shown in min-max pulse difference (Table 3).

The standard deviation of the long-term continuous RR intervals and the standard deviation of instantaneous beat-to-beat variability has not shown a clear, significant improvement. Considering changes on an individual level, these parameters show a narrowing tendency (Table 3). The decreasing trend in HRV was confirmed with the data found in our research on COPD patients (Table 3). We would like to underline that 12 patients were in a very severe parasympathetic overload. On average the four-week treatment resulted in no significant improvement in stda and stdb, but the ratio shifted into a sympathetic autonomic modulation.

As a result of the four-week rehabilitation program, the number of pairs of adjacent $\mathrm{NN}$ intervals differing by more than $50 \mathrm{~ms}$ divided by the total number of all $\mathrm{NN}$ intervals and the spectral analysis of the low-frequency/high-frequency ratio showed improvement. although these changes did not achieve clinical significance (Table 3). These results show that four weeks of rehabilitation had a favorable impact on autonomic function and overall wellbeing.

\section{Discussion}

Pulmonary rehabilitation program resulted in a clear decrease in HRV data. with a shift into sympathetic autonomic modulation. As a result of the rehabilitation program in our research, we did not find any correlation between HRV and functional markers. Although decreasing HRV is related to increased morbidity and mortality, we believe that the changes in HRV were caused by higher sympathetic stimulation, contributing to improved health-related quality of life, autonomic function and overall wellbeing.

Beat-to-beat variability in heart rate is a well-known phenomenon. Breathing, digestion, thermoregulation, but even breathing aritmia, extrusion, applying cold to the face, changes in body position and stress all lead to changes in the length of time between heartbeats; the heart rate variability (HRV) analysis measures these changes [34-36]. HRV has been used as a tool to analyze the behavior of the autonomic nervous system on the heart and to compare possible differences between healthy and unhealthy people. Decrease in HRV is related to increased morbidity and mortality [22,31]. Other studies indicate that there are changes in HRV in a list of cardiorespiratory disorders. COPD is associated with vascular remodeling that modifies the pulmonary circulation; this pathological mechanism is usually caused by hypoxia generated by the disease [35].

Reis, et al. [37] aimed to evaluate the influence of respiratory 
Citation: Szucs B, Petrekanits M, Varga J (2018) Effectiveness of a Pulmonary Rehabilitation Program on Changes in Heart Rate Variability and Physical Performance in Chronic Obstructive Pulmonary Disease. J Pulm Respir Med 8: 474. doi: 10.4172/2161-105X.1000474

Page 4 of 5

muscle strength on the magnitude of respiratory sinus arrhythmia. They assumed that respiratory muscle weakness negatively influences HRV during respiratory maneuvers in COPD. This study had a very similar protocol to ours: ECG signal and the instantaneous HR were obtained at rest in the supine position for 15 minutes while volunteers went through a respiratory sinus arrhythmia maneuver in the same position in the following order: For one minute at rest with spontaneous breathing; for four minutes while performing the respiratory sinus arrhythmia maneuver; and for one minute at rest with spontaneous breathing. As in our study, COPD patients showed evidence of impaired autonomic modulation of heart rate at rest and during respiratory sinus arrhythmia maneuver.

Both COPD and congestive heart failure (CHF) patients show alterations in autonomic modulation of heart rate at rest and during respiratory sinusal arrhythmia maneuver (RSA-M) compared with apparently healthy individuals, according to a study by Reis, et al. [37] COPD patients showed a reduction in sympathetic activity compared to the control group at rest. In our study we found similar results at the start of the four-week rehabilitation program: Lower min-max pulse difference, depressed Poincaré plot proportion and LF/HF ratio. The patients demonstrated reduced sympathetic activity; in 12 patients we found heavy parasympathetic overload. These findings indicate a possible relationship between regulatory changes of the autonomic centers and the sensitivity of chemoreceptors or respiratory pattern characterized by periodic oscillations in COPD patients.

In a recent review Mohamed, et al. [38] found that aerobic exercise training causes a significant increase in time domain HRV, but frequency domain HRV analyses were not significantly affected by aerobic exercise training. Also, baroreceptor sensitivity seems to be very low in COPD patients. causing a limited beneficial effect on autonomic function. This can be one reason for the HRV data not reaching clinical significance in our study.

In a clinical study, de Carvalho, et al. [39] found decreased stda (SD1) and stdb (SD2) in COPD patients group while the stda/stdb ratio were similar between healthy and COPD patient groups. These findings are in line with ours and also suggest a reduced HRV in patients with COPD. The reduced stda index indicates a reduction in the activity of these individuals. Compared with healthy subjects he found a collapse in the values of stdb too in patients with COPD. This indicates an overall reduction in the autonomic modulation of these individuals, which suggests global autonomic damage in COPD. Our findings show similar tendencies; both stda and stdb show loss of variability, reduced vagal control and autonomic modulation. These effects are chronic as pulmonary rehabilitation program has not resulted in clinically significant changes in stda and stdb.

De Carvalho, et al. [39] also found greater dispersion of data through the analysis of the Poincaré plot in COPD, which, again, suggests the same reduction in HRV. Van Gestel came to a similar conclusion in a study [40]. The aim was to evaluate the association between cardiac autonomic dysfunction and health-related quality of life in COPD patients. A six-minute walk test was performed by patients in a 30 $\mathrm{m}$ indoor track. Resting parasympathetic tone was independently associated with health-related quality of life. The importance of supervised exercise in subjects with COPD was highlighted since the literature shows beneficial effects on cardiac autonomic modulation. As a result of rehabilitation, concentrating mainly on physical rehabilitation through exercise, we found a strong sympathetic activity in autonomic modulation and a clear, significant improvement in most functional and quality of life markers.
Camillo, et al. [41] analyzed HRV after two exercise programs in 40 COPD patients. Two groups were created: A high-intensity, endurance exercise group and low-intensity calisthenics, breathing exercise group. The three months of high-intensity exercise training proved to have an important role in post-training cardiac autonomic function improvement in patients with COPD. This underlines the importance of further investigation of the sustained long-term effects of our fourweek rehabilitation program.

HRV is an important tool for assessing the autonomic nervous system (ANS), which has an important role in maintaining homeostasis. It is a clear predictor of the internal functions of the body, whether they are normal or pathological conditions. The correct usage can lead to characterization, evaluation and identification of problems in human development, growth and health [42].

Our study has limitations. One of them is that we focused only on patients with COPD and not other chronic respiratory diseases. Our pulmonary rehabilitation program was somewhat shorter than an eight-week program. but the total amount of work was comparable; our patients performed training two to three times per day with high intensity of load. Further research is needed to better understand the effects of training load and rehabilitation program on autonomic control of COPD patients.

\section{Conclusion}

The data collected in this study show the importance of HRV as a low-cost method for early diagnosis of cardiovascular diseases, deranged autonomic modulation, and loss of HRV concomitant with lung diseases since these diseases tend to coexist, influencing the morbidity and mortality of these patients. It is well-known that COPD patients tend to have a reduced HRV and, consequently, a deterioration of symptoms. Our study attempted to show the importance and complexity of the pulmonary rehabilitation program, as it helps to minimize the effects that COPD may have in patients.

\section{Acknowledgement}

The study protocol was approved by the Ethical Committee of the National Koranyi Institute for Pulmonology with 25/2017 registration number. The study was financed by the sources available at Deparment of Pulmonary Rehabilitation. National Koranyi Institute for Pulmonology, Budapest, Hungary.

\section{Conflict of Interest}

None of the authors reported conflict of interest

\section{References}

1. Murray CJ, Lopez AD (1997) Alternative projections of mortality and disability by cause 1990-2020. Global Burden Disease Study. Lancet 349: 1498-1504.

2. Van den Berg P, Pinsky M (2000) Systems approach to heart-lung interactions Neth J Med 57: 113-131.

3. Sarubbi B, Esposito V, Ducceschi V, Meoli I, Grella E, et al. (1997) Effect of blood gas derangement on QTc dispersion in severe chronic obstructive pulmonary disease: Evidence of an electropathy? Int J Cardiol 58: 287-292.

4. Flick MR, Block AJ (1979) Nocturnal vs. diurnal cardiac arrhythmias in patients with chronic obstructive pulmonary disease. Chest 75: 8-11.

5. Tirlapur VG, Mir MA (1982) Nocturnal hypoxemia and associated electrocardiographic changes in patients with chronic obstructive airways disease. N Engl J Med 306: 125-130.

6. Stewart AG, Waterhouse JC, Howard P (1995) The QTC interval, autonomic neuropathy and mortality in hypoxaemic COPD. Respir Med 89: 79-84.

7. Chen WL, Chen GY, Kuo CD (2006) Hypoxemia and autonomic nervous dysfunction in patients with chronic obstructive pulmonary disease. Respir Med 100: 1547-1553.

8. Chhabra SK, De S (2005) Cardiovascular autonomic neuropathy in chronic 
Citation: Szucs B, Petrekanits M, Varga J (2018) Effectiveness of a Pulmonary Rehabilitation Program on Changes in Heart Rate Variability and Physical Performance in Chronic Obstructive Pulmonary Disease. J Pulm Respir Med 8: 474. doi: 10.4172/2161-105X.1000474

Page 5 of 5

obstructive pulmonary disease. Respir Med 99: 126-133.

9. Khandoker AH, Jelinek HF, Palaniswami M (2009) Identifying diabetic patients with cardiac autonomic neuropathy by heart rate complexity analysis. Biomed Eng Online 8: 3.

10. Tereshchenko LG, Cygankiewicz I, McNitt S, Vazquez R, Bayes-Genis A, et al. (2012) Predictive value of beat-to-beat QT variability index across the continuum of left ventricular dysfunction: Competing risks of non-cardiac or cardiovascular death, and sudden or non-sudden cardiac death. Circ Arrhythm Electrophysiol 5: 719-727.

11. Fagard RH (1992) Impact of different sports and training on cardiac structure and function. Cardiol Clin 10: 241-256.

12. Quintana DS, Heathers JA, Kemp AH (2012) On the validity of using the Polar RS800 heart rate monitor for heart rate variability research. Eur J Appl Physio 112: $4179-4180$.

13. Vanderlei LC, Silva RA, Pastre CM, Azevedo FM, Godoy MF (2008) Comparison of the Polar S810i monitor and the ECG for the analysis of heart rate variability in the time and frequency domains. Braz J Med Biol Res 10: 854-859.

14. Aubert AE, Seps B, Beckers F (2003) Heart rate variability in athletes. Sports Med 33: 889-919.

15. Acharya UR, Suri JS, Spaan JAE, Krishnan SM (2007) Advances in cardiac signal processing. Berlin, New York: Springer.

16. Vatner SF, Pagani M (1976) Cardiovascular adjustments to exercise: Hemodynamics and mechanisms. Prog Cardiovasc Dis 19: 91-108.

17. Volterrani M, Scalvini S, Mazzuero G, Lanfranchi P, Colombo R, et al. (1994) Decreased heart rate variability in patients with chronic obstructive pulmonary disease. Chest 106: 1432-1437.

18. Bartels MN, Gonzalez JM, Kim W, De Meersman RE (2000) Oxygen supplementation and cardiac-autonomic modulation in COPD. Chest 118: 691 696.

19. Bartels MN, Jelic S, Gonzalez JM, Kim W (2004) Reproducibility of heart rate and blood pressure variability in patients with chronic obstructive pulmonary disease. Clin Auton Res 14: 194-196.

20. Takabatake N, Nakamura H, Minamihaba O, Inage M, Inoue S, et al. (2001) A novel pathophysiologic phenomenon in cachexic patients with chronic obstructive pulmonary disease: The relationship between the circadian rhythm of circulating leptin and the very low-frequency component of heart rate variability. Am J Respir Crit Care Med 163: 1314-1319.

21. Bartels MN, Jelic S, Ngai P, Basner RC, DeMeersman RE (2003) Highfrequency modulation of heart rate variability during exercise in patients with COPD. Chest 124: 863-869.

22. Van den Berg ME, Stricker BH, Bruselle GG, Lahousse L (2016) Chronic obstructive pulmonary disease and sudden cardiac death: A systematic review. Trends Cardiovasc Med 26: 606-613.

23. Task Force of the European Society of Cardiology and the North American Society of Pacing and Electrophysiology (1996) Heart rate variability: Standards of measurement, physiological interpretation and clinical use. Eur Heart J 17: 354-381.

24. Quanjer PH, Tammeling GJ, Cotes JE, Fabbri LM, Matthys H (2003) Lung volumes and forced ventilatory flows. Report working party standardization of lung function tests, european community for steel and coal. Official statement of the european respiratory society. Eur Respir J 16: 5-40.

25. Balke B (1963) A simple filed test for the assessment of physical fitness. Rep 63-6. Rep Civ Aeromed Res Inst US :1-8.

26. Holland AE, Spruit MA, Troosters T, Puhan MA, Pepin V, et al. (2014) An officia european respiratory society/american thoracic society technical standard: Field walking tests in chronic respiratory disease. Eur Respir J 44: 1428-1446.

27. Debouche S, Pitance L, Robert A, Liistro G, Reychler G (2016) Reliability and reproducibility of chest wall expansion measurement in young healthy adults. $J$ Manipulative Physiol Ther 39: 443-449.

28. Neumeister W, Rasche K, Maas P, Monnerjahn C, Singh B, et al. (1996) Reproducibility of computer-assisted mouth occlusion pressure measurements. Med Klin 2: 73-75.

29. Mirsky IA, Lipman E, Grinker RR (1946) Breath holding time in anxiety states Fed Proc 5: 74.

30. An KN, Chao NY, Askew LJ (1980) Hand strength measurement instruments. Arch Phys Med Rehab 61: 366-368.

31. Dias de Carvalho T, Marcelo Pastre C, Claudino Rossi R, de Abreu LC, Valent VE, et al. (2011) Geometric index of heart rate variability in chronic obstructive pulmonary disease. Rev Port Pneumol 17: 260.

32. Varga J, Porszasz J, Boda K, Casaburi R, Somfay A (2007) Supervised high intensity continuous and interval training vs. self-paced training in COPD. Respir Med 101: 2297-2304.

33. Kerti M, Balogh Z, Kelemen K, Varga JT (2018) The relationship between exercise capacity and different functional markers in pulmonary rehabilitation for COPD. Int J Chron Obstruct Pulmon Dis 13: 717-724.

34. Antônio AMS, Cardoso MA, de Abreu LC (2014) Fractal dynamics of heart rate variability: A study in healthy subjects.

35. Matarese A, Santulli G (2012) Angiogenesis in chronic obstructive pulmonary disease: A translational appraisal. Transl Med UniSa 30: 49-56.

36. Zupanic E, Zivanovic I, Kalisnik JM, Avbelj V, Lainscak M (2014) The effect of 4-week rehabilitation on heart rate variability and QTc interval in patients with chronic obstructive pulmonary disease. COPD 11: 659-669.

37. Reis MS, Sampaio LMM, Lacerda D, De Oliveira LV, Pereira GB, et al (2010) Acute effects of different levels of continuous positive airway pressure on cardiac autonomic modulation in chronic heart failure and chronic obstructive pulmonary disease. Arch Med Sci: AMS 6: 719-727.

38. Mohammed J, Derom E, VanOosterwijck J, Da Silva H, Calders P (2018) Evidence for aerobic exercise training on the autonomic function in patients with chronic obstructive pulmonary disease (COPD): A systematic review. Physiother 104: 36-45.

39. De Dias Carvalho T, Pastre CM, Rossi RC, Abreu LC, Valenti VE, et al. (2011) Índices geométricos de variabilidade da frequência cardíaca na doença pulmonar obstrutiva crônica. Rev Port Pneumol 17: 260-265.

40. Van Gestel AJ, Kohler M, Steier J, Teschler S, Russi EW, et al. (2011) Cardiac autonomic dysfunction and health-related quality of life in patients with chronic obstructive pulmonary disease. Respirol 16: 939-946.

41. Camilo CA, Vde Laburu M, Gonçalves NS, Cavalheri V, Tomasi FP, et al. (2011) Improvement of heart rate variability after exercise training and its predictors in COPD. Respir Med 105: 1054e-1062.

42. Abreu LC (2012) Heart rate variability as a functional marker of development. J Hum Growth Dev 22: 279-282. 